\title{
Graphene-based tunable coding metasurfaces in terahertz band
}

\author{
Jialin Feng ${ }^{1,{ }^{*}}$, Hongyu $\mathrm{Shi}^{1}$, Anxue Zhang ${ }^{2}$, and Zhuo $\mathrm{Xu}^{3}$ \\ ${ }^{1}$ Ministry of Education Key Laboratory for Multifunctional Materials and Structures, Xi'an Jiaotong University, Xi'an 710049, \\ PR China \\ 2 School of Electronic and Information Engineering, Xi'an Jiaotong University, Xi'an 710049, China \\ 3 The Electronic Materials Research Laboratory, Key Laboratory of the Ministry of Education, Xi'an Jiaotong University, Xi'an \\ 710049, PR China
}

Received: 13 March 2020 / Accepted: 30 July 2020

\begin{abstract}
Two graphene-based tunable coding metasurfaces are proposed for beam steering in terahertz band. The coding metasurfaces are composed of the unit cell with a sandwich-like structure, which contains the top layer of anisotropic rectangular graphene structure, intermediate dielectric layer and ground plane. The designed metasurfaces can be dynamically adjusted since the characteristics of unit cell are changed by the chemical potential of graphene. When the relaxation time and chemical potential of graphene are $0.8 \mathrm{ps}$ and $0.85 \mathrm{eV}$, respectively. The coding metasurfaces could realize beam steering in $1.30 \mathrm{THz}-1.70 \mathrm{THz}$. On the other hand, when the chemical potential of graphene is $0 \mathrm{eV}$, two metasurfaces without beam steering in this band. The designed graphene-based tunable coding metasurfaces has potential application value in the fields of terahertz communication, sensing, etc.
\end{abstract}

Keywords: Coding metasurfaces / graphene-based tunable / beam-steering / terahertz

\section{Introduction}

In recent years, terahertz science and technology has developed rapidly, and it has shown the promising application prospects in communication, imaging and nondestructive testing [1-3]. These applications of $\mathrm{THz}$ waves require not only efficient terahertz source but also terahertz devices such as polarization converter [4], phase shifter [5] etc. However, there are few materials and devices in nature that can manipulate $\mathrm{THz}$ waves effectively, which limits the development of $\mathrm{THz}$ waves.

Metasurfaces, two-dimensional metamaterials, which not only can flexibly manipulate the characteristics of EM waves such as amplitude, phase and polarization, but also have the advantages of ultra-thin, low loss and easy fabricating compared with metamaterials [6]. For traditional metamaterial, which can be called "analogy metamaterial" and described by effective permittivity and permeability. In 2014, the coding metasurfaces was proposed for digital and real-time control of EM waves compared with traditional analogy metamaterial [7]. For coding metasurface, "0" and "1" represent the unit cell with

\footnotetext{
* e-mail: fenghui@stu.xjtu.edu.cn
}

phase response " 0 " and " $\pi$ ", respectively. We could arrange " 0 " and " 1 " unit cells for different coding sequences to achieve more efficient control of EM waves.

Currently, more and more metasurfaces for control $\mathrm{THz}$ waves is presented, for example, wave plate [8], hologram [9], vector beam [10] and generation of non-linear harmonic wave [11]. However, most of these proposed terahertz metasurfaces with fixed performances after the design is completed, which limits their application. Hence, more attentions should be paid to dynamic regulate $\mathrm{THz}$ wave for multiple functions. Thus far, the common regulate methods for $\mathrm{THz}$ metasurfaces include light field regulation [12], electric field regulation [13] and temperature regulation [14].

Graphene has become one of the popular materials for tunable terahertz devices due to its unique electromagnetic properties. The conductivity of graphene can be adjusted by the chemical potential [15-17], the chemical potential of graphene can be changed by chemical doping and applying bias voltage. It is available for tunable terahertz metasurfaces to realize dynamic regulation of $\mathrm{THz}$ waves. Recently, some tunable graphene metasurfaces was proposed $[18,19]$, but most of them are narrow band, low efficiency and complicated. It is desired to design adjustable, efficient and simple tunable $\mathrm{THz}$ metasurfaces. 


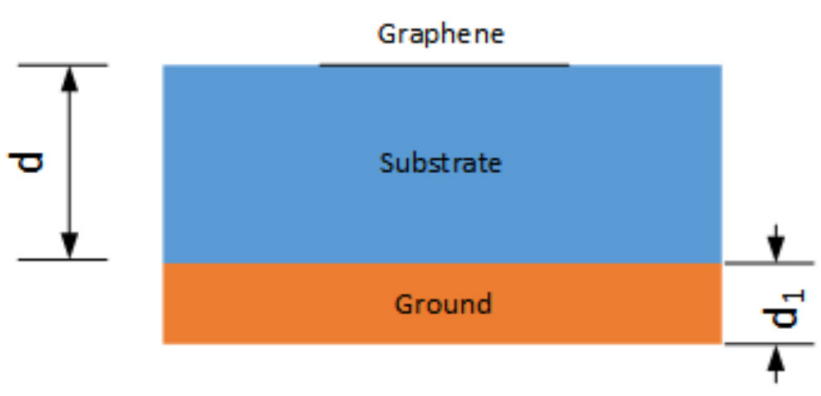

(a)

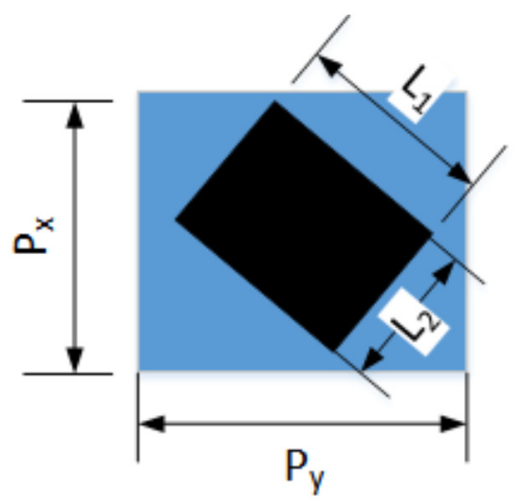

(b)

Fig. 1. (a) Side view of unit cell, (b) Top view of unit cell.

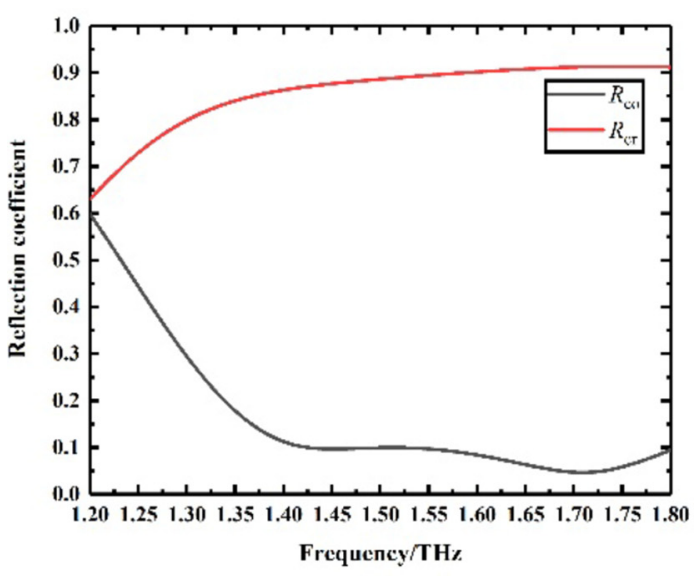

(a)

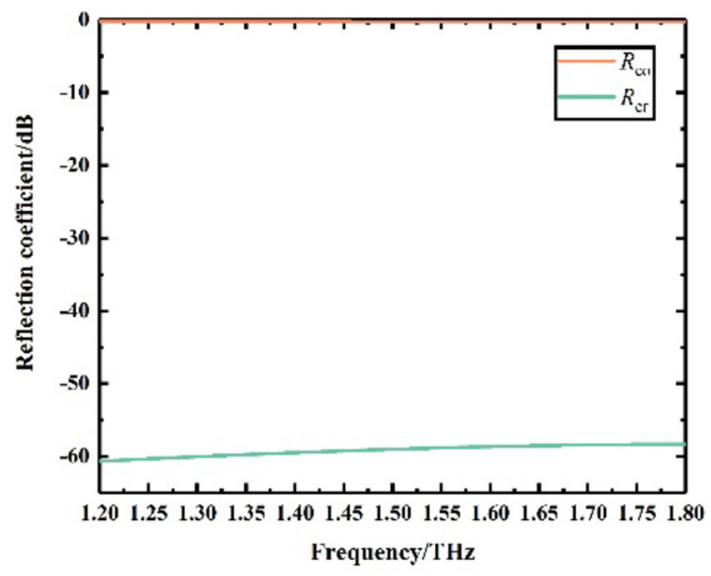

(b)

Fig. 2. The reflective characteristics of unit cell when (a) $\mu_{c}=0.85 \mathrm{eV}$, (b) $\mu_{c}=0 \mathrm{eV}$.

In this paper, we proposed two tunable coding metasurfaces based on graphene for beam steering in terahertz band. When given the relaxation time and chemical potential of graphene are $\tau=0.8 \mathrm{ps}$ and $\mu_{c}=0.85 \mathrm{eV}$. The coding metasurfaces formed by the unit cells which can achieve cross-polarization conversion from $1.30 \mathrm{THz}$ to $1.70 \mathrm{THz}$, and the amplitude of cross-polarization conversion over $90 \%$. Two metasurfaces could realize beam-steering in this band through different coding sequences. At the same time, the electromagnetic properties of unit cell are adjusted due to the change of graphene chemical potential, so the coding metasurfaces is tunable.

\section{Design of unit cell}

The structure of designed unit cell is shown in Figure 1, which could achieve cross-polarization conversion in $\mathrm{THz}$ band. The unit cell comprises of a layer of graphene, dielectric substrate and ground plate (PEC). As shown in Figure 1, the rectangular graphene rotate $45^{\circ}$ with a size of $L_{1}{ }^{*} L_{2}\left(L_{1}=32 \mu \mathrm{m}, \mathrm{L}_{2}=15 \mu \mathrm{m}\right)$ is coated on the substrate, blue parts of unit cell represent dielectric substrate whose thickness is $13 \mu \mathrm{m}$, Silicon is chosen as dielectric substrate and relative dielectric constants $\varepsilon_{r}=3.84$. The thickness of metal layer is $2 \mathrm{um}$, the period of unit cell is $P x^{*} P y$, $P x=P y=40 \mu \mathrm{m}$.

Illuminated by linear-polarized EM waves, the unit cell can convert it into cross-polarization EM waves in reflect direction. Because the impedance of graphene is influenced by the chemical potential $\mu_{c}$, so the amplitude and phase of reflected wave can regulated by adjust the $\mu_{\mathrm{c}}$. When $\mu_{c}=0.85 \mathrm{eV}, \tau=0.8 \mathrm{ps}$, the reflection cross-polarization conversion characteristics of unit cell are shown in Figure 2a. $R$ denote the amplitude of the reflect waves, $R_{c o}$ is the co-polarized reflectance of the reflected wave, $R_{c r}$ refers to the cross-polarized reflectance of the reflected wave. We can observe that high-efficiency cross-polarization conversion is realized from $1.30 \mathrm{THz}$ to $1.70 \mathrm{THz}$. The amplitude of cross-polarization conversion over $90 \%$ and co-polarization below $-10 \mathrm{~dB}$ at the same time. When we change the chemical potential of graphene Figure $2 \mathrm{~b}$ depicts the unit cell can't achieve cross-polarization conversion when $\mu_{c}=0 \mathrm{eV}$, the amplitude of crosspolarization conversion below $-55 \mathrm{~dB}$, which is approximately 0 . 


\section{Analysis and design of the coding metasurface}

Coding metasurfaces allow more flexible control of EM waves compared with traditional metasurfaces. We can realize real-time and digital control of EM waves using different coding sequences. For example, the coding sequences "01010101......" can spilt one beam into two beams in two symmetric directions. Here, "0" and "1"

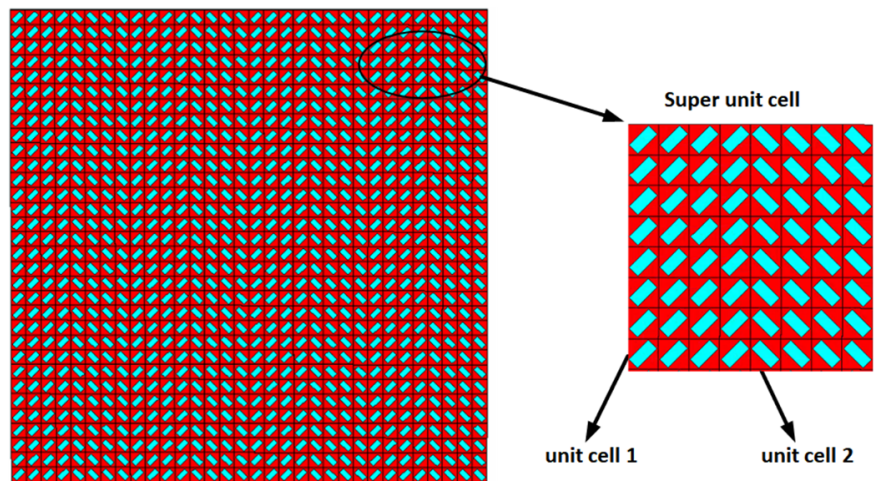

Fig. 3. The arrangement of Metasurface I and structure of Super unit cell. represent the unit cell with phase response " 0 " and " $\pi$ ", respectively. We designed two coding metasurfaces and optimized the arrangement of unit cells for beam steering. They can convert vertically incident linearpolarized $\mathrm{THz}$ waves into two/four beams propagating in two/four diagonal directions, respectively. Figure 3 depicts the arrangement of Metasurface I, it consists of $32 * 32$ unit cells, which could be regarded as $4^{*} 4$ Super unit cells. Each Super unit cell include $8 * 8$ unit cells, unit cell 1 and unit cell 2 have the same structure but rotated $90^{\circ}$. Figure 4 depicts the phase of $R_{c r}$ of unit cell 1 and unit cell 2 when the chemical potential are $0.85 \mathrm{eV}$ and $0 \mathrm{eV}$, they with $180^{\circ}$ phase difference from $1.30 \mathrm{THz}$ to $1.70 \mathrm{THz}$.

Metasurface I was simulated by CST Microwave Studio, Figures 5 and 6 show the normalized E-pattern at $1.30 \mathrm{THz}, 1.50 \mathrm{THz}, 1.70 \mathrm{THz}$ when $\mu_{c}=0.85 \mathrm{eV}$, $\mu_{c}=0 \mathrm{eV}$, respectively. From Figure 5, we can observe that EM waves reflected by Metasurface I and split into two beams that are symmetrical about the normal direction. $\theta$ denote the angle between beam and normal direction, $\theta=43.5^{\circ}, 37^{\circ}, 32^{\circ}$ at $1.30 \mathrm{THz}, 1.50 \mathrm{THz}, 1.70 \mathrm{THz}$, respectively. As shown in Figure 6, Metasurface I cannot steer beam when $\mu_{c}=0 \mathrm{eV}$. Although unit cell 1 and unit cell 2 with $180^{\circ}$ phase difference, the unit cell can not achieve cross-polarization conversion because the amplitude of $R_{c r}$ is approximately 0 .

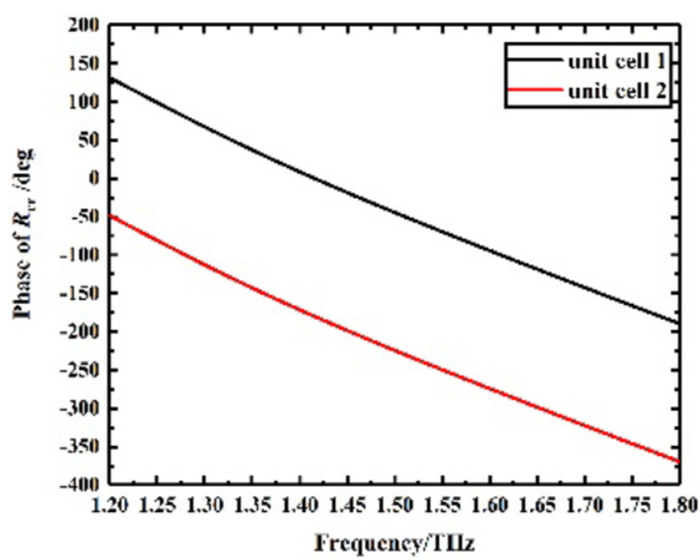

(a)

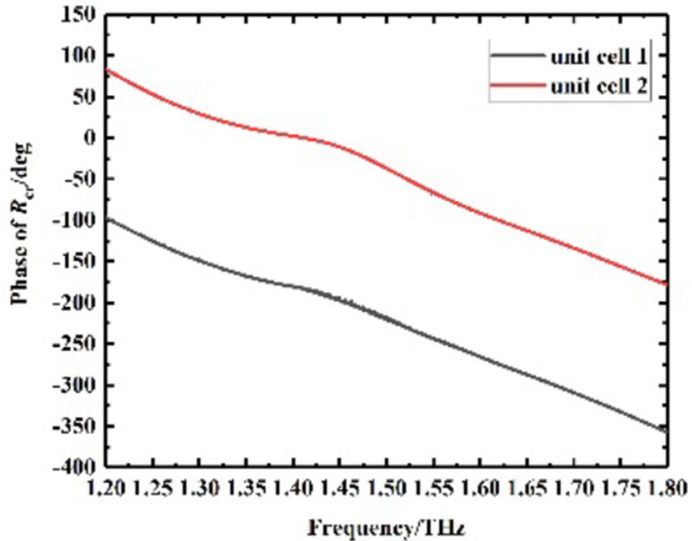

(b)

Fig. 4. The phase of $R_{c r}$ when (a) $\mu_{c}=0.85 \mathrm{eV}$, (b) $\mu_{c}=0 \mathrm{eV}$.

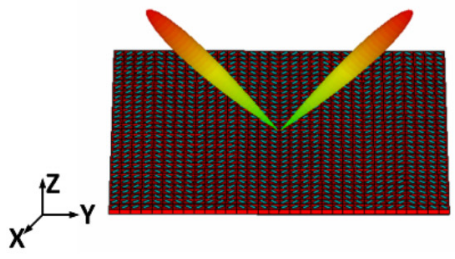

(a)

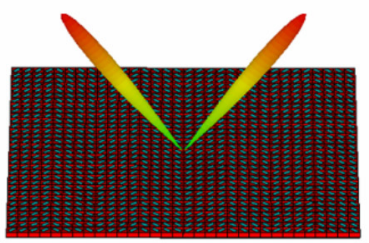

(b)

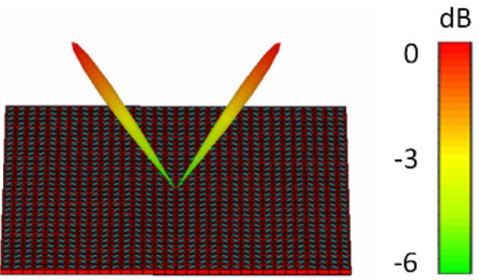

(c)

Fig. 5. Normalized E-pattern of Metasurface I at (a) $1.30 \mathrm{THz}$, (b) $1.50 \mathrm{THz}$, (c) $1.70 \mathrm{THz}$ when the chemical potential is 0.85 eV. 


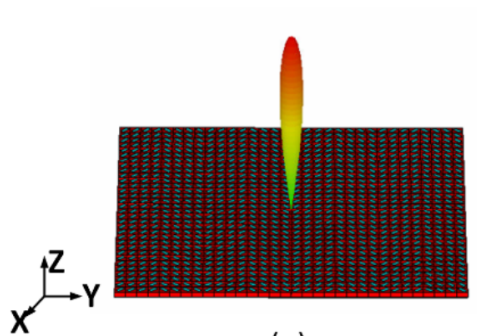

(a)

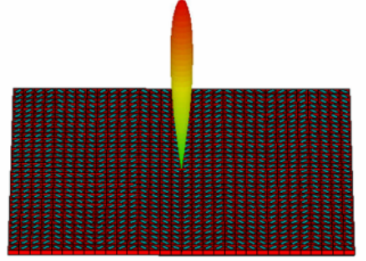

(b)

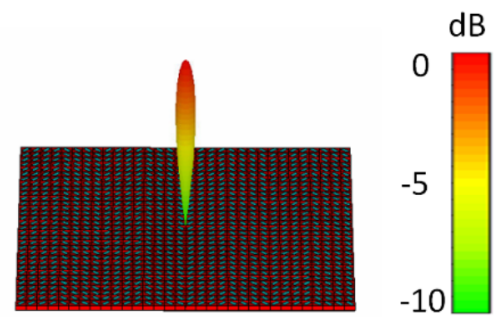

(c)

Fig. 6. Normalized E-pattern of Metasurface I at (a) $1.30 \mathrm{THz}$, (b) $1.50 \mathrm{THz}$, (c) $1.70 \mathrm{THz}$ when the chemical potential is 0 eV.

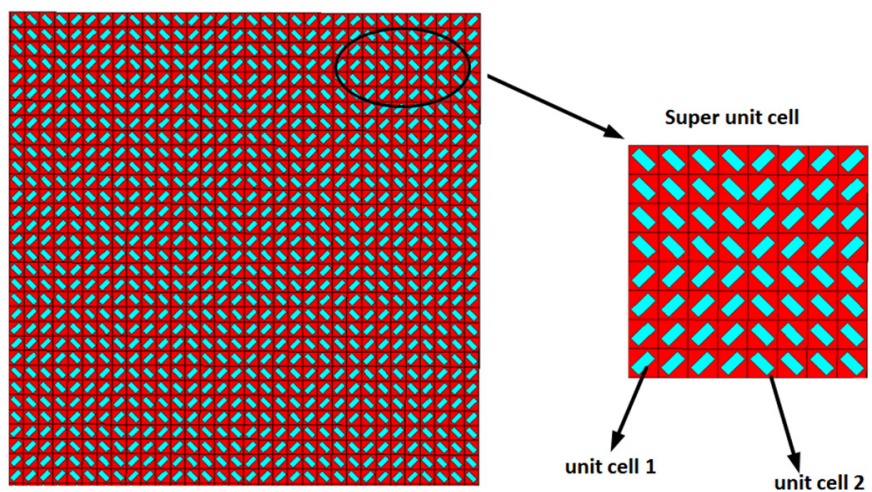

Fig. 7. The arrangement of Metasurface II and structure of its Super unit cell.

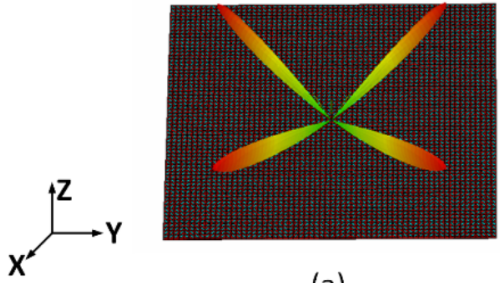

(a)

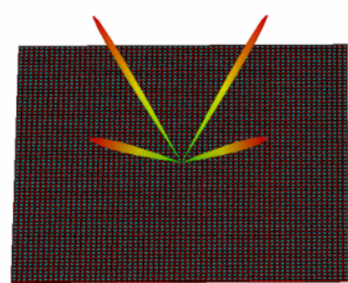

(b)

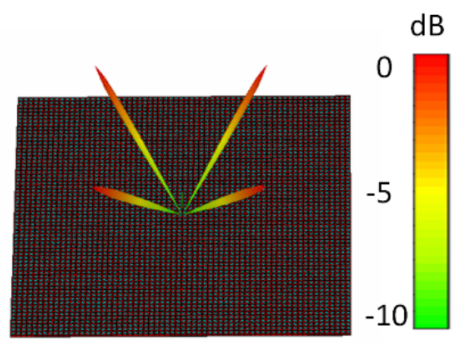

(c)

Fig. 8. Normalized E-pattern of Metasurface II at (a) $1.30 \mathrm{THz}$, (b) $1.50 \mathrm{THz}$, (c) $1.70 \mathrm{THz}$. when the chemical potential is $0.85 \mathrm{eV}$.

The Metasurface II is designed for more complex beam steering, which has same dimensions but different arrangement of unit cells compared with Metasurface I, and the Super unit cell of Metasurface II include four unit cells and composed by $8 * 8$ unit cells. The arrangement of Matasurface II is depicted in Figure 7, we can see that the four unit cells is rotate $90^{\circ}$ in turn, they satisfy the condition that every two adjacent unit cells with $180^{\circ}$ phase difference. The coding sequence of Metasurface II is "01100110.........." which can achieve the reflective beam propagating in four diagonal reflect directions.

As shown in Figure 8, when chemical potential is $0.85 \mathrm{eV}$, the reflective beam spilt into four beams in $1.30 \mathrm{THz}, 1.50 \mathrm{THz}, 1.70 \mathrm{THz} .(\theta, \varphi)$ is azimuthal angle of four beams, The angle of four scattered beams at $1.30 \mathrm{THz}$ is $\left(44^{\circ}, 70^{\circ}\right),\left(134^{\circ}, 70^{\circ}\right),\left(224^{\circ}, 70^{\circ}\right),\left(314^{\circ}, 70^{\circ}\right)$. Scattered beams at $1.50 \mathrm{THz}$ and $1.70 \mathrm{THz}$ only with different $\varphi$ values of $60^{\circ}$ and $50^{\circ}$ compared with $1.30 \mathrm{THz}$. When the chemical potential of graphene is $0 \mathrm{eV}$, the unit cell cannot realize reflection cross-polarization conversion. Metasurface II without beam steering in $1.30 \mathrm{THz} \sim 1.70 \mathrm{THz}$, it is can be observed that the reflect beam is not split into four beams from Figure 9, which is caused by the change in chemical potential of graphene.

\section{Conclusion}

In this paper, we propose two graphene-based tunable coding metasurfaces to achieve beam steering in $\mathrm{THz}$ band when the relaxation time $\tau=0.8 \mathrm{ps,} \mathrm{chemical}$ potential $\mu_{c}=0.85 \mathrm{eV}$. From $1.30 \mathrm{THz}$ to $1.70 \mathrm{THz}$, two 


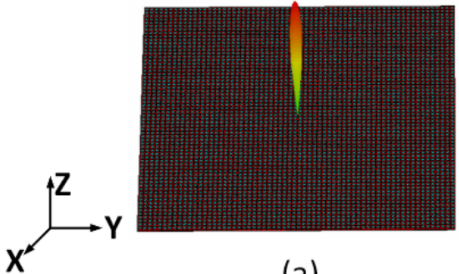

(a)

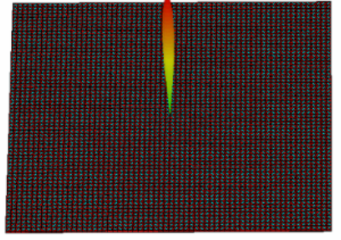

(b)

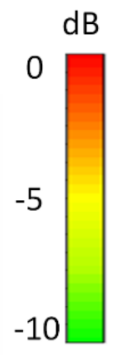

(c)

Fig. 9. Normalized E-pattern of Metasurface II at (a) $1.30 \mathrm{THz}$, (b) $1.50 \mathrm{THz}$, (c) $1.70 \mathrm{THz}$ when the chemical potential is $0 \mathrm{eV}$.

metasurfaces could convert incident linear-polarization $\mathrm{THz}$ waves into two/four reflective beam in diagonal directions. The angle of scattered beam is consistent with theoretical analysis. We have realized adjustable beam steering, when $\mu_{c}=0 \mathrm{eV}$, two coding metasurfaces without beam steering. In addition, the original periodicity is destroyed to some extent due to code and rotate the unit cells, so we cannot observe the phenomenon of beam steering at $1.80 \mathrm{THz}$.

\section{References}

1. D. Saeedkia, Handbook of Terahertz Technology for Imaging, Sensing and Communications (Woodhead Publishing, England)

2. B. Ferguson, X.C. Zhang, Nat. Mater. 1, 26 (2002)

3. Y.X. Jia, Q. Fan, Y.F. Wang, Opto-Electr. Eng. 44, 670 (2017)

4. I. Yamada, K. Takano, M. Hangyo et al., Opt. Lett. 34, 274 (2009)

5. C.Y. Chen, T.R. Tsai, C.L. Pan et al., Appl. Phys. Lett. 83, 4497 (2003)
6. N. Yu, P. Genevet, M.A. Kats et al., Science 334, 333 (2011)

7. T.J. Cui, M.Q. Qi, X. Wan et al., Light-Sci. Appl. 3, e218 (2014)

8. A. Pors, M.G. Nielsen, S.I. Bozhevolnyi, Opt. Lett. 38, 513 (2017)

9. Q. Wang, X. Zhang, Y. Xu et al., Sci. Rep. 6, 32867 (2016)

10. J.Y. Guo, X.K. Wang, J.W. He et al., Adv. Opt. Mater. 6, 1700925 (2017)

11. S. Chen, G. Li, F. Zeuner et al., Phys. Rev. Lett. 113, 033901 (2014)

12. J.W. He, X.K. Wang, Z.W. Xie et al., APL Photon. 2, 076102 (2017)

13. H.T. Chen, W.J. Padilla, J.M.O. Zide et al., Nature 444, 597 (2006)

14. E.P. Parrott, C. Han, F. Yan et al., Nanotechnology 27, 205206 (2016)

15. A.K. Geim, Science 324, 1530 (2009)

16. G.W. Hanson, J. Appl. Phys. 103, 064302 (2008)

17. A.K. Geim, K.S. Novoselov, Nat. Mater. 6, 183 (2007)

18. C. Yang, Y. Luo, J.X. Guo et al., Opt. Express 24, 16913 (2016)

19. M.L. Huang, Y.Z. Cheng, Z.Z. Cheng et al., Opt. Commun. 415, 194 (2018)

Cite this article as: Jialin Feng, Hongyu Shi, Anxue Zhang, Zhuo Xu, Graphene-based tunable coding metasurfaces in terahertz band, EPJ Appl. Metamat. 7, 5 (2020) 